\title{
Aplicações da Terapia Cognitivo-Comportamental em pacientes oncológicos: Uma
}

\section{Revisão Integrativa}

\author{
Applications of Cognitive Behavioral Therapy in Cancer Patients: An integrative review \\ Aplicaciones de la terapia cognitivo-conductual en pacientes con cáncer: Una revisión integradora
}

Recebido: 11/04/2021 | Revisado: 19/04/2021 | Aceito: 23/04/2021 | Publicado: 09/05/2021

Ítalo Souza Ferreira

ORCID: https://orcid.org/0000-0003-3879-2683 Universidade Federal de Alagoas, Brasil

E-mail: itlsouzapsi@gmail.com

Amauri dos Santos Araujo

ORCID: https://orcid.org/0000-0001-7435-5670 Universidade Federal de Alagoas, Brasil E-mail: amauriaraujo.sms@gmail.com

Rosivalda Oliveira Cajé

ORCID: https://orcid.org/0000-0001-9192-9664 Centro Universitário Tiradentes, Brasil

E-mail: rosivaldacaje@gmail.com

Andressa Pereira Lopes

ORCID: https://orcid.org/0000-0003-0789-0440 Centro Universitário Tiradentes, Brasil E-mail: aplopes.andressa@gmail.com

\begin{abstract}
Resumo
"Câncer" é um termo genérico para um grupo muito grande de doenças que podem afetar qualquer parte do corpo. Podem se dar por fatores externos como o meio ambiente, ou internos, em que a partir da formação do câncer ocorrem danos no Ácido Desoxirribonucléico celular: os termos utilizados para denominar essas alterações mais precoces até a formação da doença são oncogênese ou carcinogênese. Percebe-se a influência dos aspectos psicológicos, comportamentais e sociais na evolução e na remissão do câncer. A Terapia Cognitivo-Comportamental é uma abordagem terapêutica que busca estudar e evidenciar resultados a partir de intervenções cognitivas e/ou comportamentais; é um modelo de tratamento terapêutico estruturado e de curta duração, podendo ser aplicada em relação ao câncer e a problemáticas associadas ou decorrentes dele. Objetiva-se analisar a aplicabilidade da TCC em pacientes oncológicos. Trata-se de um estudo de revisão integrativa da literatura realizado nas bases da Biblioteca Virtual em Saúde e PubMed entre 2010 e 2020. Foram selecionados 14 estudos, contendo ensaios clínicos randomizados, intervenções que aplicaram psicoeducação, relaxamento, resolução de problemas e gerenciamento de estresse realizadas nas modalidades em grupo e/ou on-line. Além disso, identificou-se a prevalência de temas como: qualidade de vida, ansiedade, depressão, fadiga e a imagem corporal relacionados aos pacientes oncológicos. Constatou-se que a aplicação da TCC é uma abordagem eficaz para o tratamento de questões relacionados ao adoecimento, seja através de grupos, individual, on-line, presencial ou por aplicativos eletrônicos, podendo possibilitar e contribuir de forma significativa no tratamento de pessoas com câncer.
\end{abstract}

Palavras-chave: Neoplasmas; Paciente oncológico; Psico-oncologia; Terapia cognitivo-comportamental; Oncologia.

\begin{abstract}
"Cancer" is a generic term for a very large group of diseases that can affect any part of the body. They can occur due to external factors such as the environment, or internal, in which, from the formation of cancer, damage occurs in cellular deoxyribonucleic acid: the terms used to describe these earlier changes until the formation of the disease are oncogenesis or carcinogenesis. The influence of psychological, behavioral and social aspects on the evolution and remission of cancer is perceived. Cognitive-Behavioral Therapy is a therapeutic approach that seeks to study and evidence results from cognitive and/or behavioral interventions; it is a model of structured and short-term therapeutic treatment, which can be applied in relation to cancer and the problems associated with or resulting from it. The objective is to analyze the applicability of CBT in cancer patients. This is an integrative literature review study carried out on the basis of the Virtual Health Library and PubMed between 2010 and 2020. 14 studies were selected, containing randomized clinical trials, interventions that applied psychoeducation, relaxation, problem solving and stress management carried out in group and/or online modalities. In addition, the prevalence of topics such as: quality of life, anxiety, depression, fatigue and body image related to cancer patients was identified. It was found that the application of CBT is an effective approach for the treatment of issues related to illness, whether through groups,
\end{abstract}


individual, online, in person or through electronic applications, which can enable and contribute significantly to the treatment of people with cancer.

Keywords: Neoplasms; Psycho-oncology; Cognitive behavioral therapy; Oncology patient; Oncology.

\begin{abstract}
Resumen
"Cáncer" es un término genérico para un grupo muy grande de enfermedades que pueden afectar cualquier parte del cuerpo. Pueden ocurrir debido a factores externos como el medio ambiente, o internos, en los que, a partir de la formación del cáncer, se produce un daño en el ácido desoxirribonucleico celular: los términos utilizados para describir estos cambios tempranos hasta la formación de la enfermedad son oncogénesis o carcinogénesis. Se percibe la influencia de los aspectos psicológicos, conductuales y sociales en la evolución y remisión del cáncer. La terapia cognitivo-conductual es un enfoque terapéutico que busca estudiar y evidenciar los resultados de las intervenciones cognitivas y/o conductuales; es un modelo de tratamiento terapéutico estructurado y de corta duración, que puede aplicarse en relación con el cáncer y los problemas asociados o derivados del mismo. El objetivo es analizar la aplicabilidad de la TCC en pacientes con cáncer. Se trata de un estudio de revisión integradora de la literatura realizado sobre la base de la Biblioteca Virtual en Salud y PubMed entre 2010 y 2020. Se seleccionaron 14 estudios, que contienen ensayos clínicos aleatorizados, intervenciones que aplicaron psicoeducación, relajación, resolución de problemas y manejo del estrés realizadas en grupo. y / o modalidades online. Además, se identificó la prevalencia de temas como: calidad de vida, ansiedad, depresión, fatiga e imagen corporal relacionados con los pacientes con cáncer. Se encontró que la aplicación de la TCC es un enfoque eficaz para el tratamiento de cuestiones relacionadas con la enfermedad, ya sea a través de grupos, individualmente, en línea, en persona o mediante aplicaciones electrónicas, que pueden habilitar y contribuir significativamente al tratamiento de personas con cáncer.
\end{abstract}

Palabras clave: Neoplasias; Paciente con cancer; Psicooncología; Terapia de conducta cognitiva; Oncología.

\title{
1. Introdução
}

"Câncer" é um termo genérico para um grupo muito grande de doenças que podem afetar qualquer parte do corpo. Uma de suas características é o crescimento de células anormais, de modo difuso, que vão além de seus limites, com possibilidade de ser espalhadas para outras regiões corporais (metástase). Essa doença tem apresentado altas taxas de prevalência, em ambos os sexos, assim como em todas as faixas etárias, representando uma das principais causas de mortalidade (Fonseca \& Castro, 2016; Carvalho et al., 2020; WHO, 2020).

As causas do câncer são multifatoriais. Podem se dar por fatores internos, em que a partir da formação do câncer ocorrem danos no Ácido Desoxirribonucléico (DNA) celular: os termos utilizados para denominar essas alterações mais precoces até a formação da doença são oncogênese ou carcinogênese. Fatores externos presentes no meio ambiente - como radiação, exposição a produtos químicos, tabaco, alimentos e outros componentes - podem contribuir para o surgimento e a evolução da doença (Fonseca \& Castro, 2016; de Oliveira, de Moraes \& de Moura, 2016; WHO, 2020).

No que diz respeito às taxas de incidência mundial do câncer, em 2008, foram registrados 12,6 milhões de casos. Em 2018, o índice aumentou para 18,1 milhões de novos casos e, em relação aos óbitos, foram registrados 9,6 milhões. Concernente às estimativas nacionais para novos casos de câncer em 2020, de acordo com o Instituto Nacional do Câncer (Inca), estão previstos 625 mil casos. Esses dados também são esperados para os anos de 2021 e 2022. Em 2020, as previsões de novos casos para o ano de 2040 são de 29,4 milhões (Inca, 2019; de Jesus et al., 2020; WHO, 2020).

Percebe-se, então, o aumento exponencial dos diagnósticos de câncer, assim como altas estatísticas de diagnósticos na população nacional e internacional. Leva-se em consideração que, ao longo dos anos, através das práticas de assistência oncológica, foi possível perceber a influência dos aspectos psicológicos, comportamentais e sociais na evolução e na remissão do câncer. O surgimento de sinais físicos, como mudança da imagem corporal e medo das mutilações, provoca diversas reações emocionais no paciente oncológico. Dessa forma, a doença, muitas vezes, causa a perda da autonomia, ameaça a expectativa de vida e os planos futuros (Gaspar, 2011; de Freitas Aguiar, 2019; Vilaça \& Campos, 2020).

Algumas reações emocionais identificadas, como angústia, ansiedade, alívio, esperança, desesperança, desamparo, apreensão, medo e diversos sentimentos e emoções, podem surgir no percurso do enfrentamento do câncer. Diante disso, faz-se necessário o uso de intervenções psicológicas para auxiliar os pacientes a lidar e manejar suas emoções e sentimentos de uma 
forma mais funcional, com orientações, esclarecimentos, escuta psicológica e suporte psicológico, além de outros recursos para o enfrentamento da doença. Técnicas cognitivas e comportamentais podem ser utilizadas, visando à melhor qualidade de vida, visão de si mesmo, da doença e do futuro (Gaspar, 2011; de Freitas Aguiar, 2019; José et al., 2020; Vilaça \& Campos, 2020).

A Terapia Cognitivo-Comportamental (TCC) é uma abordagem terapêutica que busca estudar e evidenciar resultados benéficos a partir de intervenções cognitivas e/ou comportamentais, e que também pode ser aplicada em relação ao câncer e a problemáticas associadas ou decorrentes dele. Alguns resultados sobre essas intervenções da TCC relacionada ao câncer podem ser encontrados nos estudos de Lourenção, Santos Junior e Luiz (2010), Rafihi-Ferreira e Soares (2012), Torres, Pereira e Monteiro (2012), Oliveira, Moraes e Moura (2016), Stang e Gomes (2017), Oliveira, de Moraes, de Moura e Ambrósio (2018).

A TCC é um modelo de tratamento terapêutico estruturado e de curta duração criado por Aaron Beck por volta da década de 1960. Ela parte da perspectiva de que o pensamento influencia o sentimento, que, por sua vez, influencia os comportamentos. Ademais, a modificação destes pensamentos, denominados disfuncionais, pode possibilitar a consequente modificação do comportamento do cliente, utilizando tanto técnicas cognitivas quanto comportamentais (Beck, 2014; Peron \& Sartes, 2015).

Esta abordagem baseia-se na aliança terapêutica sólida, na formulação em desenvolvimento constante dos problemas dos pacientes e no avanço individual de cada um deles. Enfatiza, ainda, a colaboração e a participação ativa do paciente, bem como a valorização do presente, e objetiva-se ensiná-lo a ser seu próprio terapeuta (Wright, Basco \& Thase, 2008; Beck, 2014).

A TCC, no tratamento de pacientes oncológicos, demonstrou resultados favoráveis, tanto individualmente quanto em grupos. Identificou-se que essa abordagem auxiliou no manejo para a gestão do stress, na melhora em relação ao enfrentamento da doença e na percepção de apoio social. Entre os recursos citados, existe a reestruturação cognitiva, o enfrentamento e as técnicas de relaxamento como os mais utilizados concomitantemente no tratamento de pacientes oncológicos (Deep, Leal e Patrão, 2014; Stang \& Gomes, 2017).

Num estudo realizado por Lourenção, Santos Junior e Luiz (2010), cujo objetivo foi realizar o levantamento de pesquisas que utilizaram intervenções cognitivo-comportamentais, observou-se que a TCC demonstrou resultados consideráveis em relação às várias problemáticas que podem surgir ao longo da vida dos pacientes oncológicos e em seus familiares durante o tratamento. Dentre eles, podemos citar: insônia, manejo dos efeitos adversos/sequelas do tratamento, manejo da dor, transtornos sexuais, sobrepeso/obesidade, tratamento de comorbidades psiquiátricas como ansiedade e depressão, queixas psicossociais e outras problemáticas.

Diante do exposto, surge a necessidade de responder à seguinte questão disparadora: qual a aplicabilidade da Terapia Cognitivo-Comportamental em pacientes oncológicos? Diante do exposto, o objetivo da pesquisa foi analisar a aplicabilidade da Terapia Cognitivo-Comportamental em pacientes oncológicos.

\section{Metodologia}

Trata-se de um estudo de revisão integrativa da literatura. Esse tipo de estudo permite que os pesquisadores construam uma síntese de trabalhos para a realização de uma ampla abordagem metodológica referente às revisões, partindo da inclusão de estudos experimentais e não experimentais para uma compreensão maior acerca do fenômeno analisado (Whittemore \& Knafl, 2005; Souza, Silva \& Carvalho, 2010; dos Santos Araujo et al., 2018).

Nesse tipo de estudo, foi necessário percorrer seis etapas distintas para a execução metodológica: 1. Identificação do tema e seleção da hipótese ou questão de pesquisa; 2. Estabelecimento de critérios para inclusão e exclusão de estudos/amostragem ou busca na literatura; 3. Definição das informações a serem extraídas dos estudos 
selecionados/categorização dos estudos; 4. Avaliação dos estudos incluídos na revisão integrativa; 5. Interpretação dos resultados; 6. Apresentação da revisão/síntese do conhecimento (Whittemore \& Knafl, 2005; Mendes, Silveira \& Galvão, 2008; Souza, Silva \& Carvalho, 2010; Botelho, de Almeida Cunha \& Macedo, 2011; dos Santos Araujo et al., 2018).

Desse modo, a coleta de informações foi feita através das bases de dados Biblioteca Virtual em Saúde (BVS) e PubMed, a partir da realização de cruzamento dos descritores "Neoplasms" "Oncology patient" , "Psycho-Oncology" , "Cognitive Behavioral Therapy" e "Oncology", de acordo com formulários específicos para pesquisa em cada base, utilizando a combinação com o booleano AND, conforme apresenta a Tabela 1.

Tabela 1 - Processo de seleção dos artigos após leitura integral do estudo. Maceió - AL, 2020.

\begin{tabular}{|c|c|c|c|c|}
\hline DeCS & $\begin{array}{c}\text { Base } \\
\text { de } \\
\text { Dados }\end{array}$ & $\begin{array}{c}\text { Artigos } \\
\text { Encontrados }\end{array}$ & $\begin{array}{l}\text { Artigos } \\
\text { Excluídos }\end{array}$ & Amostra \\
\hline $\begin{array}{c}\text { Neoplasms } \\
\text { AND } \\
\text { Psycho-Oncology } \\
\text { AND } \\
\text { Cognitive Behavioral Therapy } \\
\text { AND } \\
\text { Oncology patient }\end{array}$ & BVS & 25 & 24 & 1 \\
\hline $\begin{array}{c}\text { Neoplasms } \\
\text { AND } \\
\text { Cognitive Behavioral Therapy } \\
\text { AND } \\
\text { Oncology patient }\end{array}$ & BVS & 146 & 142 & 4 \\
\hline $\begin{array}{c}\text { Psycho-Oncology AND } \\
\text { Cognitive Behavioral Therapy }\end{array}$ & PubMed & 71 & 70 & 1 \\
\hline $\begin{array}{c}\text { Oncology patient } \\
\text { AND } \\
\text { Cognitive Behavioral Therapy } \\
\text { AND } \\
\text { Oncology }\end{array}$ & PubMed & 420 & 412 & 8 \\
\hline
\end{tabular}

\footnotetext{
${ }^{1}$ Neoplasmas.

${ }^{2}$ Paciente oncológico.

${ }^{3}$ Psico-Oncologia.

${ }^{4}$ Terapia Cognitivo-Comportamental.

${ }^{5}$ Oncologia.

Fonte: Autores (2020).
}

Para esse refinamento, foram adotados os seguintes critérios de inclusão: artigos científicos com ano de publicação no recorte temporal de 2010 a 2020; artigos em português e em inglês, disponibilizados na íntegra e gratuitamente e que tratem da Terapia Cognitivo-Comportamental Beckiana, bem como, abordem todos os tipos de cânceres e em todas as faixas etárias. Foram adotados os seguintes critérios de exclusão: livros, teses, monografias, dissertações, cartas ao leitor, artigos duplicados e textos não científicos e que apresentem problemas de acesso ao artigo indexado nas bases de dados selecionada, artigos com intervenções voltadas apenas aos familiares dos pacientes oncológicos, artigos com estudos clínicos inacabados, artigos com intervenções voltadas a sobreviventes do câncer/pós-câncer e artigos com intervenções voltadas a profissionais de saúde. 


\section{Resultados e Discussão}

Em uma busca inicial dos termos, utilizando as estratégias de pesquisa, foram encontrados 662 estudos. Foram excluídos 648 estudos, por não preencherem alguns dos critérios de inclusão estabelecidos na metodologia deste trabalho. Foram selecionados 14 estudos após realização de análise conforme os critérios estabelecidos, a partir da leitura inicial do título e do resumo do estudo e, posteriormente, sua leitura na íntegra.

O Quadro 1 apresenta a produção científica acerca do tema, com características de distribuição quanto ao título do artigo, autores, ano de publicação, objetivo, tipo de estudo, intervenções utilizadas, modalidade, tipo e síntese dos principais resultados. 
Quadro 1 - Título, ano, objetivo, tipo de estudo, intervenções utilizadas, modalidade, tipo e síntese dos resultados incluídos na amostra. Maceió - AL, 2020.

\begin{tabular}{|c|c|c|c|c|c|c|c|c|}
\hline $\begin{array}{l}\text { TÍTULO DO } \\
\text { ARTIGO }\end{array}$ & AUTOR & ANO & OBJETIVO & $\begin{array}{l}\text { TIPO DE } \\
\text { ESTUDO }\end{array}$ & INTERVENÇÕES UTILIZADAS & $\begin{array}{l}\text { MODALI- } \\
\text { DADE }\end{array}$ & TIPO & $\begin{array}{c}\text { SÍNTESE DOS PRINCIPAIS } \\
\text { RESULTADOS }\end{array}$ \\
\hline $\begin{array}{l}\text { Psychosocial } \\
\text { Interventions } \\
\text { for Advanced } \\
\text { Cancer Patients: } \\
\text { A Systematic } \\
\text { Review. }\end{array}$ & $\begin{array}{l}\text { Teo, } \\
\text { Krishnan e } \\
\text { Lee }\end{array}$ & 2019 & $\begin{array}{l}\text { Fornecer uma visão } \\
\text { geral ampla das } \\
\text { intervenções } \\
\text { psicossociais não } \\
\text { médicas testadas na } \\
\text { última década. }\end{array}$ & $\begin{array}{l}\text { Revisão } \\
\text { Sistemáti- } \\
\text { ca. }\end{array}$ & $\begin{array}{l}\text { Psicoeducação; Definição de metas; } \\
\text { Resolução de problemas; Reavaliação } \\
\text { cognitiva; Treinamento de } \\
\text { relaxamento/atenção plena; Treinamento } \\
\text { de habilidades de enfrentamento; } \\
\text { Gerenciamento de estresse e atividade } \\
\text { planejamento/ritmo. }\end{array}$ & $\begin{array}{l}\text { Presencialmente } \\
\text {, por telefone ou } \\
\text { on-line; } \\
\text { modalidades } \\
\text { mistas. }\end{array}$ & $\begin{array}{l}\text { Sessões } \\
\text { indivi- } \\
\text { duais, } \\
\text { diádicas } \\
\text { ou em } \\
\text { grupo. }\end{array}$ & $\begin{array}{l}\text { A maioria dos estudos }(60 \%) \text { relatou melhora, } \\
\text { incluindo qualidade de vida e sintomas como } \\
\text { dor, fadiga, falta de ar e angústia relacionada } \\
\text { à falta de ar. }\end{array}$ \\
\hline $\begin{array}{l}\text { Cancer!? I } \\
\text { Don't Have } \\
\text { Time for That: } \\
\text { Impact of a } \\
\text { Psychosocial } \\
\text { Intervention for } \\
\text { Young Adults } \\
\text { with Cancer. }\end{array}$ & $\begin{array}{l}\text { Aubin et } \\
\text { al. }\end{array}$ & 2019 & $\begin{array}{l}\text { Avaliar o impacto } \\
\text { de uma intervenção } \\
\text { cognitivo- } \\
\text { comportamental } \\
\text { adaptada para } \\
\text { atender às } \\
\text { necessidades e } \\
\text { questões } \\
\text { psicossociais da } \\
\text { população de } \\
\text { adolescentes e } \\
\text { adultos jovens com } \\
\text { câncer. }\end{array}$ & $\begin{array}{l}\text { Estudo } \\
\text { Randomi- } \\
\text { zado } \\
\text { Controlado }\end{array}$ & $\begin{array}{l}\text { Resolução de problemas/Tomada de } \\
\text { decisões; Gerenciamento de medo; } \\
\text { Técnica de relaxamento breve; } \\
\text { Automonitoramento; Pausas } \\
\text { comportamentais; Reenquadramento } \\
\text { cognitivo; reformulação cognitiva, } \\
\text { atenção plena sexual, jogos de } \\
\text { comunicação e questionamento } \\
\text { auto/introspectivo e gerenciamento de } \\
\text { tempo de qualidade. }\end{array}$ & $\begin{array}{l}\text { Realizada por } \\
\text { meio do Skype } \\
\text { ou sessões } \\
\text { presenciais. }\end{array}$ & $\begin{array}{l}\text { Sessões } \\
\text { indivi- } \\
\text { duais. }\end{array}$ & $\begin{array}{l}\text { Melhorias de bem-estar social e sexual. } \\
\text { Melhorias significativas pré e pós-intervenção } \\
\text { foram obtidas nos domínios do humor geral e } \\
\text { QVRS e em domínios específicos de } \\
\text { ansiedade e estima sexual. Pós-intervenção } \\
\text { para melhora do acompanhamento foi obtida } \\
\text { no domínio da autoeficácia relacionada à } \\
\text { doença. } \\
\text { Intervenção igualmente eficaz } \\
\text { presencialmente ou on-line. }\end{array}$ \\
\hline $\begin{array}{l}\text { Randomized } \\
\text { Trial of a } \\
\text { Tailored } \\
\text { Cognitive- } \\
\text { Behavioral } \\
\text { Therapy Mobile } \\
\text { Application for } \\
\text { Anxiety in } \\
\text { Patients with } \\
\text { Incurable } \\
\text { Cancer. }\end{array}$ & Greer et al. & 2019 & $\begin{array}{l}\text { Testar a eficácia de } \\
\text { um aplicativo móvel } \\
\text { (app) de Terapia } \\
\text { Cognitivo- } \\
\text { Comportamental } \\
\text { (TCC) para tratar a } \\
\text { ansiedade em } \\
\text { pacientes com } \\
\text { câncer incurável. }\end{array}$ & $\begin{array}{l}\text { Ensaio } \\
\text { Clínico } \\
\text { Randomi- } \\
\text { zado }\end{array}$ & $\begin{array}{l}\text { Introdução ao aplicativo móvel e } \\
\text { psicoeducação; Treinamento de } \\
\text { relaxamento; Planejamento e Ritmo de } \\
\text { Atividades; Comunicação para solução de } \\
\text { problemas; Ficar Presente; Criação de } \\
\text { novos pensamentos; Resumo. }\end{array}$ & $\begin{array}{l}\text { Aplicativo de } \\
\text { TCC em } \\
\text { aparelho } \\
\text { eletrônico. }\end{array}$ & $\begin{array}{l}\text { O artigo } \\
\text { não } \\
\text { especifi- } \\
\text { cou se foi } \\
\text { individu- } \\
\text { almente } \\
\text { ou em } \\
\text { grupo. }\end{array}$ & $\begin{array}{l}\text { Os participantes do aplicativo móvel TCC } \\
\text { relataram uma maior redução nos sintomas de } \\
\text { ansiedade na subescala HADS. } \\
\text { Os que completaram as atividades do } \\
\text { aplicativo móvel de TCC relataram melhorias } \\
\text { significativas em todas as medidas de } \\
\text { autorrelato para ansiedade, sintomas de } \\
\text { depressão e QV em comparação com os que } \\
\text { não concluíram as atividades do aplicativo } \\
\text { móvel de TCC. } \\
\text { O subgrupo de pacientes com ansiedade basal }\end{array}$ \\
\hline
\end{tabular}




\begin{tabular}{|c|c|c|c|c|c|c|c|c|}
\hline & & & & & & & & $\begin{array}{l}\text { grave teve maiores reduções nos sintomas de } \\
\text { ansiedade com o aplicativo móvel TCC em } \\
\text { comparação com o programa de educação em } \\
\text { saúde. }\end{array}$ \\
\hline $\begin{array}{l}\text { Preliminary } \\
\text { Results From a } \\
\text { Randomized } \\
\text { Controlled } \\
\text { Study for an } \\
\text { App-Based } \\
\text { Cognitive } \\
\text { Behavioral } \\
\text { Therapy } \\
\text { Program for } \\
\text { Depression and } \\
\text { Anxiety in } \\
\text { Cancer Patients. }\end{array}$ & Ham et al. & 2019 & $\begin{array}{l}\text { Desenvolver e } \\
\text { avaliar a eficácia de } \\
\text { um programa de } \\
\text { Terapia Cognitivo- } \\
\text { Comportamental } \\
\text { baseado em } \\
\text { aplicativo para } \\
\text { depressão e } \\
\text { ansiedade em } \\
\text { pacientes com } \\
\text { câncer. }\end{array}$ & $\begin{array}{l}\text { Ensaio } \\
\text { Clínico } \\
\text { Randomi- } \\
\text { zado. }\end{array}$ & $\begin{array}{l}\text { Psicoeducação; Ativação } \\
\text { comportamental; Treinamento de } \\
\text { relaxamento; Reestruturação cognitiva; } \\
\text { Resolução de problemas. }\end{array}$ & $\begin{array}{l}\text { Aplicativo de } \\
\text { TCC em } \\
\text { aparelho } \\
\text { eletrônico. }\end{array}$ & $\begin{array}{l}\text { O artigo } \\
\text { não } \\
\text { especifi- } \\
\text { cou se foi } \\
\text { indi- } \\
\text { vidual- } \\
\text { mente ou } \\
\text { em grupo }\end{array}$ & $\begin{array}{l}\text { O programa de TCC baseado em aplicativo } \\
\text { foi considerado eficaz na redução da } \\
\text { depressão e da ansiedade entre pacientes com } \\
\text { câncer. Os resultados positivos deste estudo } \\
\text { também servem como evidência de apoio para } \\
\text { a eficácia da TCC para depressão e ansiedade } \\
\text { em geral. } \\
\text { O programa mostrou eficácia em uma ampla } \\
\text { gama de pacientes com câncer com vários } \\
\text { tipos e estágios de câncer. }\end{array}$ \\
\hline $\begin{array}{l}\text { Cognitive } \\
\text { Behavioral } \\
\text { Therapy for } \\
\text { Treatment- } \\
\text { Related Fatigue } \\
\text { in Chronic } \\
\text { Myeloid } \\
\text { Leukemia } \\
\text { Patients on } \\
\text { Tyrosine Kinase } \\
\text { Inhibitors: A } \\
\text { Mixed-Method } \\
\text { Study. }\end{array}$ & Poort et al. & 2019 & $\begin{array}{l}\text { Explorar a } \\
\text { viabilidade e a } \\
\text { eficácia da TCC } \\
\text { para a fadiga } \\
\text { relacionada à } \\
\text { terapia direcionada. }\end{array}$ & $\begin{array}{l}\text { Experiment } \\
\text { os de caso } \\
\text { único } \\
\text { replicados } \\
\text { (SCEs) e } \\
\text { entrevistas. }\end{array}$ & $\begin{array}{l}\text { Psicoeducação; Formulação de objetivos } \\
\text { de tratamento; exposição; Desafiar as } \\
\text { crenças disfuncionais. }\end{array}$ & $\begin{array}{l}\text { O artigo não } \\
\text { especificou se } \\
\text { era presencial } \\
\text { ou on-line. }\end{array}$ & $\begin{array}{l}\text { Sessões } \\
\text { Indivi- } \\
\text { duais. }\end{array}$ & $\begin{array}{l}\text { Diminuição na direção esperada para a } \\
\text { gravidade da fadiga em todos os quatro } \\
\text { participantes, mas os resultados gerais não } \\
\text { indicaram um efeito de intervenção } \\
\text { significativo. Os participantes relataram } \\
\text { melhorias qualitativas; foram mais capazes de } \\
\text { lidar com a fadiga, experimentaram menos } \\
\text { interferência da fadiga no funcionamento } \\
\text { diário e a maioria relatou melhora na } \\
\text { qualidade de vida. }\end{array}$ \\
\hline $\begin{array}{l}\text { A Randomised } \\
\text { Controlled Trial } \\
\text { of a Brief } \\
\text { Cognitive } \\
\text { Behavioural } \\
\text { Intervention for }\end{array}$ & $\begin{array}{l}\text { Stefanopou } \\
\text { lou, } \\
\text { Yousaf, } \\
\text { Grunfeld e } \\
\text { Hunter }\end{array}$ & 2015 & $\begin{array}{l}\text { Avaliar os efeitos de } \\
\text { uma intervenção de } \\
\text { Terapia Cognitivo- } \\
\text { Comportamental de } \\
\text { autoajuda guiada } \\
\text { (TCC) na }\end{array}$ & $\begin{array}{l}\text { Ensaio } \\
\text { Clínico } \\
\text { Randomi- } \\
\text { zado. }\end{array}$ & $\begin{array}{l}\text { Informações sobre as causas de HFNS, o } \\
\text { modelo de TCC e fatores que afetam } \\
\text { HFNS, como a modificação de gatilhos; } \\
\text { TCC para pensamentos e crenças } \\
\text { excessivamente negativos sobre HFNS e } \\
\text { estratégias comportamentais, usando }\end{array}$ & $\begin{array}{l}\text { O artigo não } \\
\text { especificou se } \\
\text { era presencial } \\
\text { ou on-line. E } \\
\text { parte da } \\
\text { intervenção }\end{array}$ & $\begin{array}{l}\text { Sessões } \\
\text { em grupo } \\
\text { e parte } \\
\text { indivi- } \\
\text { dual. }\end{array}$ & $\begin{array}{l}\text { A TCC parece ser uma intervenção segura e } \\
\text { eficaz para ajudar os pacientes com câncer de } \\
\text { próstata submetidos à ADT a controlar a } \\
\text { incômoda HFNS. No pós-tratamento, o grupo } \\
\text { de TCC relatou HFNS menos problemática e } \\
\text { menos frequente do que o grupo de TAU, com }\end{array}$ \\
\hline
\end{tabular}


Research, Society and Development, v. 10, n. 5, e33410514941, 2021

(CC BY 4.0) | ISSN 2525-3409 | DOI: http://dx.doi.org/10.33448/rsd-v10i5.14941

\begin{tabular}{|c|c|c|c|c|c|c|c|c|}
\hline $\begin{array}{l}\text { Men Who Have } \\
\text { Hot Flushes } \\
\text { Following } \\
\text { Prostate Cancer } \\
\text { Treatment } \\
\text { (MANCAN). }\end{array}$ & & & $\begin{array}{l}\text { classificação de } \\
\text { problemas de ondas } \\
\text { de calor, suores } \\
\text { noturnos e } \\
\text { frequência (HFNS), } \\
\text { humor e qualidade } \\
\text { de vida relacionada } \\
\text { à saúde em } \\
\text { pacientes } \\
\text { submetidos à terapia } \\
\text { de privação de } \\
\text { andrógenos (ADT). }\end{array}$ & & $\begin{array}{l}\text { respiração ritmada e relaxamento; } \\
\text { Estratégias para gerenciar o sono e NS. }\end{array}$ & $\begin{array}{l}\text { através de } \\
\text { chamadas } \\
\text { telefônicas. }\end{array}$ & & $\begin{array}{l}\text { reduções clinicamente relevantes. Às } 32 \\
\text { semanas, o grupo de TCC manteve sua } \\
\text { melhora, mas as diferenças de grupo não } \\
\text { atingiram significância, em grande parte por } \\
\text { causa de alguma melhora no grupo TAU. } \\
\text { No entanto, a TCC foi eficaz no ajuste das } \\
\text { características basais e independentemente do } \\
\text { IMC, nível educacional ou tipo de câncer. No } \\
\text { entanto, a intervenção não reduziu } \\
\text { significativamente o humor ou a qualidade de } \\
\text { vida. }\end{array}$ \\
\hline $\begin{array}{l}\text { Cognitive } \\
\text { Behavioral } \\
\text { Therapy in } \\
\text { Breast Cancer } \\
\text { Patients: A } \\
\text { Feasibility } \\
\text { Study of an } 8 \\
\text { Week } \\
\text { Intervention for } \\
\text { Tumor } \\
\text { Associated } \\
\text { Fatigue } \\
\text { Treatment. }\end{array}$ & $\begin{array}{l}\text { Eichler et } \\
\text { al. }\end{array}$ & 2015 & $\begin{array}{l}\text { Desenvolver um } \\
\text { protocolo simples, } \\
\text { reprodutível e curto } \\
\text { ( } 8 \text { semanas) para o } \\
\text { suporte da Terapia } \\
\text { Cognitivo- } \\
\text { Comportamental de } \\
\text { pacientes com } \\
\text { fadiga associada a } \\
\text { tumor. }\end{array}$ & $\begin{array}{l}\text { Estudo de } \\
\text { viabilidade } \\
\text { de interven- } \\
\text { ção. }\end{array}$ & $\begin{array}{l}\text { Técnicas de relaxamento; Técnicas de } \\
\text { enfrentamento e gerenciamento de } \\
\text { recursos. }\end{array}$ & $\begin{array}{l}\text { Ambulatório. O } \\
\text { artigo não } \\
\text { especificou se } \\
\text { era presencial } \\
\text { ou on-line. }\end{array}$ & $\begin{array}{l}\text { Sessões } \\
\text { em grupo. }\end{array}$ & $\begin{array}{l}\text { Este estudo mostrou que uma intervenção em } \\
\text { grupo de Terapia Cognitivo-Comportamental } \\
\text { altamente estruturada produzirá melhorias } \\
\text { significativas nos níveis de fadiga associados } \\
\text { a tumores de pacientes com câncer de mama } \\
\text { após apenas } 8 \text { semanas. } \\
\text { Depois de completar } 8 \text { sessões de grupo, } \\
\text { apenas } 7 \text { pacientes mantiveram uma } \\
\text { pontuação de diagnóstico TAF patológico. } \\
\text { Essa é uma redução de aproximadamente } 70 \% \\
\text { e por si só já mostra o benefício desse } \\
\text { programa. Além disso, uma diminuição } \\
\text { estatisticamente significativa na pontuação } \\
\text { média de ansiedade e depressão pode ser } \\
\text { mostrada usando o questionário HADS. Esta } \\
\text { análise mostrou uma redução de } 33 \% \text { nos } \\
\text { sintomas de ansiedade e 57\% de redução e } \\
\text { depressão. }\end{array}$ \\
\hline $\begin{array}{l}\text { A Review of the } \\
\text { Effectiveness of } \\
\text { Cognitive- } \\
\text { Behavioral } \\
\text { Group Therapy } \\
\text { on the }\end{array}$ & $\begin{array}{l}\text { Faraji, } \\
\text { Mahdavi, } \\
\text { Samkhaniy } \\
\text { an, Asadi e } \\
\text { Dezhkam }\end{array}$ & 2015 & $\begin{array}{l}\text { Examinar a eficácia } \\
\text { da Terapia } \\
\text { Cognitivo- } \\
\text { Comportamental em } \\
\text { grupo na redução de } \\
\text { preocupações com a }\end{array}$ & $\begin{array}{l}\text { Estudo } \\
\text { quase } \\
\text { experimenta } \\
1 \text { com pré- } \\
\text { teste-pós- } \\
\text { teste e }\end{array}$ & $\begin{array}{l}\text { Relaxamento; Treinamento para regular a } \\
\text { excitação; Técnicas comportamentais } \\
\text { como exposição imaginária e real. }\end{array}$ & $\begin{array}{l}\text { O artigo não } \\
\text { especificou se } \\
\text { era on-line ou } \\
\text { presencial. }\end{array}$ & $\begin{array}{l}\text { Sessões } \\
\text { em grupo. }\end{array}$ & $\begin{array}{l}\text { Impactos significativos na melhoria da } \\
\text { preocupação com a imagem corporal em } \\
\text { pacientes com câncer de mama. As técnicas } \\
\text { cognitivas e comportamentais ajudaram os } \\
\text { pacientes a confrontar gradualmente partes de } \\
\text { seu corpo que causavam desconforto usando }\end{array}$ \\
\hline
\end{tabular}


Research, Society and Development, v. 10, n. 5, e33410514941, 2021

(CC BY 4.0) | ISSN 2525-3409 | DOI: http://dx.doi.org/10.33448/rsd-v10i5.14941

\begin{tabular}{|c|c|c|c|c|c|c|c|c|}
\hline $\begin{array}{l}\text { Reduction of } \\
\text { Body Image } \\
\text { Concern in } \\
\text { Patients with } \\
\text { Breast Cancer. }\end{array}$ & & & $\begin{array}{l}\text { imagem corporal } \\
\text { em pacientes com } \\
\text { câncer de mama. }\end{array}$ & $\begin{array}{l}\text { grupo } \\
\text { controle. }\end{array}$ & & & & $\begin{array}{l}\text { técnicas de relaxamento e reduzindo } \\
\text { gradualmente sua tensão, dor e ansiedade, e } \\
\text { assim obter uma sensação de satisfação e } \\
\text { integridade. }\end{array}$ \\
\hline $\begin{array}{l}\text { Cognitive } \\
\text { Behavioural } \\
\text { Therapy for } \\
\text { Depression in } \\
\text { Multiple } \\
\text { Endocrine } \\
\text { Neoplasia Type } \\
\text { IIB: A 1-Year } \\
\text { Follow-Up. }\end{array}$ & $\begin{array}{l}\text { Mejía- } \\
\text { Castrejón e } \\
\text { Landa- } \\
\text { Ramírez }\end{array}$ & 2014 & $\begin{array}{l}\text { Avaliar o impacto } \\
\text { da TCC na } \\
\text { depressão maior e } \\
\text { na qualidade de vida } \\
\text { de um paciente com } \\
\text { câncer. }\end{array}$ & $\begin{array}{l}\text { Relato de } \\
\text { Caso. }\end{array}$ & $\begin{array}{l}\text { Psicoeducação sobre depressão e MEN } \\
\text { IIB; Ativação comportamental; } \\
\text { Reestruturação de pensamentos } \\
\text { negativos; Treinamento em habilidades } \\
\text { sociais; Assertividade. }\end{array}$ & $\begin{array}{l}\text { Sessões } \\
\text { presenciais. }\end{array}$ & $\begin{array}{l}\text { Sessões } \\
\text { indivi- } \\
\text { duais. }\end{array}$ & $\begin{array}{l}\text { Houve a redução da depressão e ajudou a } \\
\text { melhorar a qualidade de vida e foi } \\
\text { considerada uma intervenção aceitável para o } \\
\text { paciente, com resultados positivos contínuos } 1 \\
\text { ano após a última sessão de psicoterapia. }\end{array}$ \\
\hline $\begin{array}{l}\text { Managing Body } \\
\text { Image } \\
\text { Difficulties of } \\
\text { Adult Cancer } \\
\text { Patients: } \\
\text { Lessons from } \\
\text { Available } \\
\text { Research. }\end{array}$ & $\begin{array}{l}\text { Fingeret, } \\
\text { Teo e } \\
\text { Epner }\end{array}$ & 2014 & $\begin{array}{l}\text { Delinear uma } \\
\text { abordagem prática } \\
\text { para avaliar e tratar } \\
\text { preocupações } \\
\text { comuns com a } \\
\text { imagem corporal de } \\
\text { pacientes adultos } \\
\text { com câncer. }\end{array}$ & $\begin{array}{l}\text { Revisão } \\
\text { Bibliográ- } \\
\text { fica }\end{array}$ & $\begin{array}{l}\text { Psicoeducação; Gerenciamento de } \\
\text { estresse; Resolução de problemas; } \\
\text { Reenquadramento cognitivo e } \\
\text { treinamento de habilidades de } \\
\text { comunicação; Estabelecimento de metas; } \\
\text { Reestruturação cognitiva; } \\
\text { Dessensibilização sistemática. }\end{array}$ & $\begin{array}{l}\text { O artigo não } \\
\text { especificou se } \\
\text { era } \text { on-line ou } \\
\text { presencial. }\end{array}$ & $\begin{array}{l}\text { Sessões } \\
\text { com } \\
\text { casais ou } \\
\text { em } \\
\text { grupos. }\end{array}$ & $\begin{array}{l}\text { Todos os } 4 \text { estudos de intervenção baseados } \\
\text { em TCC relataram melhora nos resultados da } \\
\text { imagem corporal após a intervenção: } 3 \\
\text { estudos relataram diferenças estatisticamente } \\
\text { significativas em comparação com os } \\
\text { controles, enquanto um estudo relatou } \\
\text { melhorias na imagem corporal que não foram } \\
\text { estatisticamente significativas. Para os } 2 \\
\text { estudos que realizaram avaliações de } \\
\text { acompanhamento, as melhorias nos resultados } \\
\text { da imagem corporal foram mantidas em } 6 \\
\text { meses e } 12 \text { meses de acompanhamento. }\end{array}$ \\
\hline $\begin{array}{l}\text { Anxiety and } \\
\text { Depression in } \\
\text { Oncology } \\
\text { Patients: A } \\
\text { Longitudinal } \\
\text { Study of A } \\
\text { Screening, } \\
\text { Assessment and } \\
\text { Psychosocial }\end{array}$ & $\begin{array}{l}\text { Thalén- } \\
\text { Lindström, } \\
\text { Larsson, } \\
\text { Glimelius } \\
\text { e } \\
\text { Johansson }\end{array}$ & 2013 & $\begin{array}{l}\text { Investigar a } \\
\text { viabilidade da } \\
\text { triagem sistemática } \\
\text { para ansiedade e } \\
\text { depressão em uma } \\
\text { população } \\
\text { heterogênea de } \\
\text { pacientes } \\
\text { oncológicos no }\end{array}$ & $\begin{array}{l}\text { Desenho de } \\
\text { grupo de } \\
\text { controle } \\
\text { histórico } \\
\text { prospecti- } \\
\text { vo. }\end{array}$ & $\begin{array}{l}\text { Focado no problema e orientado para o } \\
\text { objetivo. A lição de casa; Novas } \\
\text { estratégias ou aceitação; Avaliação dos } \\
\text { objetivos. }\end{array}$ & $\begin{array}{l}\text { Sessões } \\
\text { presenciais. }\end{array}$ & $\begin{array}{l}\text { Sessões } \\
\text { indivi- } \\
\text { duais. }\end{array}$ & $\begin{array}{l}\text { A TCC individual foi utilizada em cerca de } \\
15 \% \text { (grupo de intervenção) e } 2 \% \text { (grupo de } \\
\text { cuidados padrão), respectivamente. Com essa } \\
\text { baixa utilização, não é surpreendente que não } \\
\text { houvesse efeitos mensuráveis da intervenção } \\
\text { quando analisada em todo o grupo de } \\
\text { pacientes. Os resultados da avaliação e da } \\
\text { intervenção de suporte foram negativos, uma } \\
\text { vez que não foram observadas diferenças em }\end{array}$ \\
\hline
\end{tabular}


Research, Society and Development, v. 10, n. 5, e33410514941, 2021

(CC BY 4.0) | ISSN 2525-3409 | DOI: http://dx.doi.org/10.33448/rsd-v10i5.14941

\begin{tabular}{|c|c|c|c|c|c|c|c|c|}
\hline $\begin{array}{l}\text { Support } \\
\text { Intervention. }\end{array}$ & & & $\begin{array}{l}\text { momento de sua } \\
\text { primeira visita a um } \\
\text { departamento de } \\
\text { oncologia. }\end{array}$ & & & & & $\begin{array}{l}\text { qualquer escala e em nenhum momento entre } \\
\text { os grupos. }\end{array}$ \\
\hline $\begin{array}{l}\text { A Pilot } \\
\text { Randomized } \\
\text { Controlled Trial } \\
\text { of Brief } \\
\text { Cognitive- } \\
\text { Behavioral } \\
\text { Therapy for } \\
\text { Anxiety in } \\
\text { Patients with } \\
\text { Terminal } \\
\text { Cancer. }\end{array}$ & Greer et al. & 2012 & $\begin{array}{l}\text { Examinar a } \\
\text { viabilidade e a } \\
\text { eficácia potencial da } \\
\text { TCC breve para } \\
\text { reduzir a ansiedade } \\
\text { em pacientes com } \\
\text { câncer terminal. }\end{array}$ & $\begin{array}{l}\text { Ensaio } \\
\text { piloto } \\
\text { randomi- } \\
\text { zado } \\
\text { controlado. }\end{array}$ & $\begin{array}{l}\text { Definição de metas e educação sobre } \\
\text { ansiedade; Treinamento de relaxamento; } \\
\text { Planejamento e ritmo de atividades. } \\
\text { Também foram utilizadas estratégias de } \\
\text { enfrentamento e reforçar habilidades para } \\
\text { controlar os sintomas relacionados ao } \\
\text { câncer; prática de habilidades e tarefas de } \\
\text { casa. }\end{array}$ & $\begin{array}{l}\text { Presencial e/ou } \\
\text { por telefone, } \\
\text { visitas } \\
\text { domiciliares ou } \\
\text { on-line. }\end{array}$ & $\begin{array}{l}\text { Sessões } \\
\text { indivi- } \\
\text { duais. }\end{array}$ & $\begin{array}{l}\text { Efeitos benéficos da terapia na redução dos } \\
\text { sintomas de ansiedade e na melhoria de certos } \\
\text { aspectos da qualidade de vida ao longo do } \\
\text { tempo. A TCC breve parece viável e } \\
\text { clinicamente benéfica para reduzir o } \\
\text { sofrimento relacionado à ansiedade em } \\
\text { pacientes com câncer que estão enfrentando } \\
\text { um diagnóstico terminal. } \\
\text { Os pacientes atribuídos à TCC relataram } \\
\text { reduções significativamente maiores nos } \\
\text { sintomas de ansiedade no HADS e IES. } \\
\text { Os sintomas de depressão autorrelatados na } \\
\text { subescala de HADS-Depressão diminuíram da } \\
\text { avaliação inicial para a avaliação pós- } \\
\text { tratamento em toda a amostra. } \\
\text { Um exame das subescalas do FACT-G } \\
\text { revelou que os participantes que receberam } \\
\text { TCC relataram pior bem-estar físico ao longo } \\
\text { do tempo, mas melhora emocional e bem- } \\
\text { estar funcional. }\end{array}$ \\
\hline $\begin{array}{l}\text { Treating } \\
\text { Prolactinoma } \\
\text { and Psychosis: } \\
\text { Medication and } \\
\text { Cognitive } \\
\text { Behavioural } \\
\text { Therapy. }\end{array}$ & $\begin{array}{l}\text { Nieman et } \\
\text { al. }\end{array}$ & 2011 & $\begin{array}{l}\text { Descrever a opção } \\
\text { de TCC adicional } \\
\text { no tratamento de } \\
\text { pacientes com } \\
\text { prolactinoma e } \\
\text { psicose. }\end{array}$ & $\begin{array}{l}\text { Relato de } \\
\text { caso. }\end{array}$ & $\begin{array}{l}\text { Empirismo colaborativo e diálogo } \\
\text { socrático; Questionamento; Desafiar e } \\
\text { reestruturar suas crenças de poder; } \\
\text { Descoberta guiada; Testes } \\
\text { comportamentais. }\end{array}$ & $\begin{array}{l}\text { Foram } \\
\text { realizadas } 15 \\
\text { sessões } \\
\text { presenciais. }\end{array}$ & $\begin{array}{l}\text { Sessões } \\
\text { indivi- } \\
\text { duais. }\end{array}$ & $\begin{array}{l}\text { O PSYRATS foi usado para avaliar as } \\
\text { alucinações antes e depois do tratamento com } \\
\text { a TCC. Três fatores na escala de classificação } \\
\text { de alucinações auditivas PSYRATS: um fator } \\
\text { de características emocionais (quantidade e } \\
\text { grau de conteúdo negativo das vozes, } \\
\text { quantidade e intensidade de angústia), um } \\
\text { fator de características físicas (frequência, } \\
\text { duração, localização e volume de as vozes) e } \\
\text { um fator de interpretação cognitiva (crenças } \\
\text { sobre vozes, perturbação da vida diária e } \\
\text { controle). As pontuações em todos os três }\end{array}$ \\
\hline
\end{tabular}


Research, Society and Development, v. 10, n. 5, e33410514941, 2021

(CC BY 4.0) | ISSN 2525-3409 | DOI: http://dx.doi.org/10.33448/rsd-v10i5.14941

\begin{tabular}{|c|c|c|c|c|c|c|c|c|}
\hline & & & & & & & & $\begin{array}{l}\text { fatores diminuíram após o tratamento, com a } \\
\text { diminuição mais forte na pontuação do fator } \\
\text { de interpretação cognitiva. }\end{array}$ \\
\hline $\begin{array}{l}\text { A Randomised } \\
\text { Controlled Trial } \\
\text { of a Cognitive } \\
\text { Behavioural } \\
\text { Intervention for } \\
\text { Women Who } \\
\text { Have } \\
\text { Menopausal } \\
\text { Symptoms } \\
\text { Following } \\
\text { Breast Cancer } \\
\text { Treatment } \\
\text { (MENOS 1): } \\
\text { Trial Protocol. }\end{array}$ & $\begin{array}{l}\text { Mann, } \\
\text { Smith, } \\
\text { Hellier e } \\
\text { Hunter }\end{array}$ & 2011 & $\begin{array}{l}\text { Avaliar a eficácia da } \\
\text { TCC em grupo no } \\
\text { alívio de HF/NS em } \\
\text { mulheres que } \\
\text { fizeram tratamento } \\
\text { para câncer de } \\
\text { mama, comparando } \\
\text { a TCC em grupo e } \\
\text { os cuidados } \\
\text { habituais. }\end{array}$ & $\begin{array}{l}\text { Ensaio } \\
\text { clínico } \\
\text { randomi- } \\
\text { zado e } \\
\text { controlado. }\end{array}$ & $\begin{array}{l}\text { Discutir gatilhos de HF/NS e maneiras de } \\
\text { modificá-los; Introdução ao relaxamento } \\
\text { de corpo inteiro; Monitorar e modificar } \\
\text { gatilhos; Monitorar pensamentos, } \\
\text { sentimentos e reações comportamentais a } \\
\text { HF/NS; Praticar reações mais úteis; } \\
\text { Discutir estratégias comportamentais para } \\
\text { melhorar a qualidade do sono; Respiração } \\
\text { ritmada; Gerenciamento de estresse. }\end{array}$ & $\begin{array}{l}\text { O artigo não } \\
\text { especificou se } \\
\text { era } \text { on-line ou } \\
\text { presencial. }\end{array}$ & $\begin{array}{l}\text { Sessões } \\
\text { em grupo. }\end{array}$ & $\begin{array}{l}\text { Redução média de } 49 \% \text { na classificação do } \\
\text { problema, bem como uma redução } \\
\text { significativa na frequência autorrelatada e } \\
\text { melhorias significativas na qualidade do sono } \\
\text { e na qualidade de vida, reduções promissoras } \\
\text { na classificação e na frequência de problemas } \\
\text { de HF/NS nessas mulheres. }\end{array}$ \\
\hline
\end{tabular}

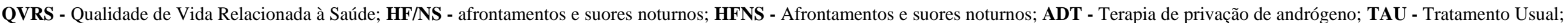

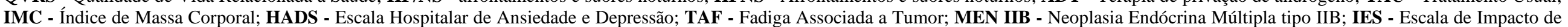

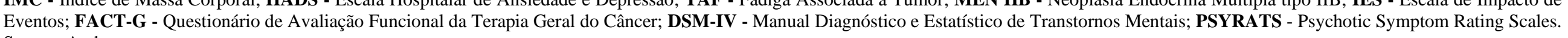
Source: Authors. 
Verificou-se a predominância de estudos do tipo ensaios clínicos randomizados (42,84\%), de intervenções que aplicaram psicoeducação $(57,12 \%)$, relaxamento $(64,26 \%)$, resolução de problemas $(28,56 \%)$ e gerenciamento de estresse $(21,42 \%)$. Houve a predominância de estudos que utilizaram a modalidade em grupo (35,7\%) e/ou on-line (42,84\%). Além disso, identificou-se a prevalência de temas como: qualidade de vida (57,12\%), ansiedade (42,84\%), depressão (42,84\%), fadiga $(21,42 \%)$ e a imagem corporal $(14,28 \%)$, relacionados aos pacientes oncológicos.

Após a análise dos estudos, optou-se por separar a apresentação dos resultados em duas grandes categorias: a) Impactos da TCC em pacientes oncológicos e b) Intervenções em TCC com pacientes oncológicos.

\subsection{Impactos da TCC em Pacientes Oncológicos}

A Terapia Cognitivo-Comportamental apresentou resultados de diminuição da ansiedade e aumento da qualidade de vida nos estudos realizados por Greer et al. (2012) e Mejía-Castrejón e Landra-Ramírez (2014), corroborando os dados encontrado por Aubin et al. (2019).

Ainda sobre a ansiedade, os estudos realizados por Aubin et al. (2019) e Ham et al. (2019) obtiveram dados significativos em relação ao uso da TCC em formato on-line, por meio de aplicativos móveis, com melhora em relação à ansiedade nos grupos da intervenção.

Em se tratando da questão da depressão, constatou-se que o estudo de Greer et al. (2012) apresenta contribuições consideráveis na redução dos sintomas depressivos, da mesma forma que os estudos de Mejía-Castrejón e Landra-Ramírez (2014) e Ham et al. (2019) demonstram que a TCC foi útil na redução da depressão. Um dado interessante sobre o estudo de Ham et al. (2019) é que os achados foram coletados em uma grande variedade de estágios e tipos de câncer.

Além disso, nota-se que, nos estudos, a TCC apresentou impacto positivo ao ser aplicada no tratamento de alucinações decorrentes do tratamento do prolactinoma (que contribuiu para o surgimento de sintomas psicóticos graves), em que utilizaram a escala PSYRATS , foram encontradas redução nos fatores - fator de características emocionais; fator de características físicas e fator de interpretação cognitiva - que compõem as medidas da escala; apresentou-se redução maior no fator de interpretação cognitiva, em que evidencia-se a diminuição das alucinações auditivas, condição está expressa por Nieman et al. (2011).

Na pesquisa de Faraji, Mahdavi, Samkhaniyan, Asadi e Dezhkam (2015), realizada por meio de estudo quase experimental com pré-teste-pós-teste e grupo controle, evidenciou-se o impacto significativo da TCC aplicada através de sessões em grupo na melhoria da atenção com a imagem corporal em pacientes com câncer de mama, possibilitando que pudessem confrontar gradualmente partes de seu corpo que causavam desconforto.

O estudo de Greer et al. (2012) encontrou, através de uma intervenção baseada em TCC breve, realizada por meio de um ensaio clínico randomizado, uma melhora emocional e bem-estar funcional, avaliados através das subescalas do FACT-G. O estudo de Aubin et al. (2019) também reitera alguns dados encontrados em Greer et al. (2012), em que se observaram melhorias relacionadas a bem-estar social, sexual, humor geral e autoeficácia relacionada à doença.

Em contrapartida, Thalén-Lindström, Larsson, Glimelius e Johansson (2013) não conseguiram observar diferenças significativas em ansiedade, depressão e qualidade de vida, a partir do uso dos instrumentos aplicados (HADS e EORTC QLQC30 - European Organization for Research and Treatment of Cancer Core Quality of Life Questionnaire) para avaliar os impactos da TCC entre os grupos estudados, mesmo sendo reavaliados em diferentes momentos durante a pesquisa. No estudo de Greer et al. (2012), em que os participantes que foram submetidos a tratamento com a TCC, no que se refere ao aspecto da qualidade de vida, foi relatado pior bem-estar relacionado ao aspecto físico dos pacientes. 
Research, Society and Development, v. 10, n. 5, e33410514941, 2021

(CC BY 4.0) | ISSN 2525-3409 | DOI: http://dx.doi.org/10.33448/rsd-v10i5.14941

Para compreender as informações apresentadas a respeito das poucas contribuições na aplicação da TCC, precisa-se levar em consideração que a amostragem do grupo de intervenção dos estudos citados não foi representativa estatisticamente para a população-alvo, devido às limitações e/ou ao objetivo da própria pesquisa (Greer et al., 2012; Thalén-Lindström, Larsson, Glimelius \& Johansson, 2013). Nota-se que, mesmo nos estudos que evidenciaram dados positivos, também é preciso verificar o impacto dessas intervenções em populações estatísticas maiores que sejam representativas.

\subsection{Intervenções em TCC com Pacientes Oncológicos}

A psicoeducação foi uma das intervenções mais citadas nos estudos analisados (57,12\%). Fingeret, Teo e Epner (2014), Teo, Krishnan e Lee (2019) e Greer et al. (2019) utilizaram a psicoeducação, mas não mencionaram sobre o que foi psicoeducado e como essa intervenção foi executada, exceto em Greer et al. (2019), que utilizaram a psicoeducação através de um aplicativo como recurso. Já no estudo de Poort et al. (2019), realizou-se a psicoeducação sobre o modelo cognitivocomportamental da fadiga relacionada ao câncer.

É importante pontuar que, no estudo de Stefanopoulou, Yousaf, Grunfeld e Hunter (2015), foram disponibilizadas informações sobre as causas de Afrontamentos e Suores Noturnos (HFNS), o modelo de TCC e fatores que afetam HFNS, mas que em nenhum momento foi utilizado o termo psicoeducação, apesar de entender-se que essas informações também são uma forma de psicoeducar.

Outra intervenção realizada foi o relaxamento (64,26\%). Autores como Mann, Smith, Hellier e Hunter (2011), Stefanopoulou, Yousaf, Grunfeld e Hunter (2015), Teo, Krishnan e Lee (2019) e Greer et al. (2019) mostraram evidências positivas das contribuições do uso dessa intervenção em pacientes oncológicos, mas não detalharam a forma de aplicação. Corroborando com esses resultados, o estudo de Eichler et al. (2015) também enfatizou o relaxamento, especificado pelos autores como de 10 a 15 minutos realizando exercícios de relaxamento e, junto com eles, introduzindo técnicas de autoconsciência.

Em relação ao uso da intervenção baseada em resolução de problemas $(28,56 \%)$, as revisões bibliográficas de Fingeret, Teo e Epner (2014) e Teo, Krishnan e Lee (2019) apresentaram uma amostra respectiva de 4 e 20 estudos baseados em intervenções que utilizaram a resolução de problemas. Não foi especificado como ocorreu o uso desta intervenção.

Intervenções baseadas em gerenciamento de estresse $(21,42 \%)$ mostraram-se frequentes e positivas em vários estudos (Mann, Smith, Hellier \& Hunter, 2011; Fingeret, Teo \& Epner, 2014; Teo, Krishnan \& Lee, 2019). De acordo com Mann, Smith, Hellier e Hunter (2011), foi utilizada uma série de exercícios interativos e discussões para aplicar essa intervenção. Autores como Fingeret, Teo e Epner (2014) e Teo, Krishnan e Lee (2019) também encontraram, em suas revisões bibliográficas, o uso do gerenciamento de estresse.

Em se tratando das modalidades de aplicação da TCC, percebe-se que, nos estudos incluídos nesta revisão, houve um maior número na modalidade grupal (Mann, Smith, Hellier \& Hunter, 2011; Fingeret, Teo \& Epner, 2014; Eichler et al., 2015; Stefanopoulou, Yousaf, Grunfeld \& Hunter, 2015; Teo, Krishnan \& Lee, 2019) e com a aplicação on-line (Greer et al., 2019; Teo, Krishnan \& Lee, 2019).

Os estudos de Fingeret, Teo e Epner (2014), Eichler et al. (2015) e Stefanopoulou, Yousaf, Grunfeld e Hunter (2015) não especificaram se a intervenção em grupo foi realizada on-line ou presencialmente. Verifica-se também que os estudos que usaram intervenções na modalidade on-line foram publicados nos últimos cinco anos e pode-se presumir/hipotetizar que seja uma promissora área de pesquisas e estudos futuros (Teo, Krishnan \& Lee, 2019; Greer et al., 2019).

Diante disso, entende-se o motivo da prevalência do uso de intervenções cognitivas e comportamentais, como 
psicoeducação, relaxamento, resolução de problemas e gerenciamento de estresse, respondendo, assim, à questão disparadora deste estudo sobre as aplicações da TCC em pacientes oncológicos como uma forma de abordagem terapêutica eficaz para o tratamento de questões relacionadas ao adoecimento oncológico, pensando em como a sua aplicação, seja através de grupos, individual, on-line, presencial ou até mesmo por aplicativos eletrônicos, pode possibilitar e contribuir de forma significativa no tratamento de pessoas com câncer.

Além disso, outro ponto que merece ser enfatizado diz respeito às dificuldades percebidas para o estudo dessa população clínica, pela própria limitação de locomoção e/ou da condição de saúde, assim como a ampla variedade de tipos de câncer e de faixas etárias em que pode se manifestar.

Cabe pontuar também a falta de especificação e detalhamento das intervenções, modalidades e aplicação dos recursos da TCC nos pacientes oncológicos, ficando evidente, principalmente no Quadro 1, em que se apresenta o relato de que alguns estudos não especificaram se a modalidade foi on-line ou presencial e se a aplicação foi individual, diádica e/ou em grupo. Assim como observado, no que se refere à coluna de intervenções, alguns estudos não adotaram a nomenclatura amplamente utilizada para descrever a intervenção realizada.

\section{Considerações Finais}

Para entender a questão da TCC e sua aplicação em pacientes com câncer, buscou-se, neste estudo, analisar a aplicabilidade da Terapia Cognitivo-Comportamental em pacientes oncológicos. Foi possível obter resultados a partir de estudos primários e secundários, por se tratar de uma revisão integrativa. Através dos resultados obtidos, percebe-se a eficácia da abordagem aplicada em pacientes oncológicos.

Nota-se que houve uma recorrência de temas nos estudos incluídos, como ansiedade, depressão, qualidade de vida, fadiga e imagem corporal, e que essa frequência pode ser justificada pela própria evolução e pelos desdobramentos relacionados à doença oncológica em si, que pode acarretar muitas reações ansiogênicas, desencadear sintomas depressivos e, consequentemente, contribuir para as alterações na qualidade de vida desses pacientes oncológicos.

A respeito das limitações encontradas neste estudo, é válido pontuar que buscou, aqui, usar apenas duas bases de dados para a seleção dos estudos e que é necessário que pesquisas futuras ampliem as bases de dados exploradas, de modo a possibilitar incluir a maior quantidade de estudos científicos disponíveis relacionados à temática. Destaca-se também que este estudo teve como foco a TCC baseada nas premissas de Aaron Beck e que é interessante que se façam pesquisas evidenciando o uso de terapias cognitivas de outras vertentes teóricas ou mesmo de terceira onda.

Outra limitação deste estudo diz respeito a que, devido ao foco não serem sobreviventes do câncer ou mesmo os familiares dos pacientes oncológicos, não foram incluídos estudos com intervenções em TCC voltadas a esses públicos, mostrando, assim, uma possível linha de pesquisas futuras para avaliar a aplicabilidade e as contribuições da terapia cognitiva. Espera-se que a presente revisão integrativa possa auxiliar os profissionais que trabalham com pacientes oncológicos, assim como pesquisadores dessa temática, e ainda estudantes e familiares e até os próprios pacientes oncológicos, acerca das contribuições que a TCC pode apresentar relacionadas à sua aplicação com câncer.

\section{Referências}

Aubin, S., Rosberger, Z., Hafez, N., Noory, M. R., Perez, S., Lehmann, S., \& Kavan, P. (2019). Cancer!? I don't have time for that: impact of a psychosocial intervention for young adults with cancer. Journal of adolescent and young adult oncology, 8(2), 172-189.

Botelho, L. L. R., de Almeida Cunha, C. C., \& Macedo, M. (2011). O método da revisão integrativa nos estudos organizacionais. Gestão e sociedade, 5(11), $121-136$. 
Research, Society and Development, v. 10, n. 5, e33410514941, 2021

(CC BY 4.0) | ISSN 2525-3409 | DOI: http://dx.doi.org/10.33448/rsd-v10i5.14941

Beck, J. S. (2014). Introdução à Terapia Cognitivo-Comportamental. Em Beck, J. S., Terapia Cognitivo-Comportamental. Artmed Editora.

Beck, J. S. (2014). Conceituação Cognitiva. Em Beck, J. S., Terapia Cognitivo-Comportamental. Artmed Editora.

Carvalho, T. V., Nakashima, S. S., Correia, T. L. B. V., Dias, S. B., Silva, M. A., dos Santos, R. C., \& Pena, H. P. (2020). Eficácia da quimioterapia nos cuidados paliativos em pacientes oncológicos: uma revisão integrativa. Research, Society and Development, 9(11), e68791110267-e68791110267.

Deep, C. N., Leal, I., \& Patrão, I. (2014). Avaliação da intervenção cognitivo-comportamental em gestão do stress em pacientes com fadiga oncológica, em radioterapia. Saúde e Sociedade, 23, 293-301.

de Jesus, D. L. D. S., da Silva, B. F., Carnevalli, C., \& da Cunha Bellinati, N. V. (2020). Cuidados paliativos oncológicos: percepção das equipes de estratégia de saúde da família. Research, Society and Development, 9(10), e4659108797-e4659108797.

de Oliveira, C. P., de Moraes, M. A., \& de Moura, T. C. (2016). Intervenções cognitivo- comportamentais para manejo de estresse em pacientes oncológicos: uma revisão sistemática. Universitas: Ciências da Saúde, 14(2).

de Oliveira, C. P., de Moraes, M. A., de Moura, T. C., \& Ambrósio, D. M. (2018). Intervenções cognitivas e comportamentais para manejo de insônia em pacientes oncológicos. Psicologia Revista, 27(1), 111-128.

dos Santos Araujo, A., Fidélis, E. P. B., da Silva, A. S. D., de Lira, L. B. S., Pessoa, I. R., Gomes, W. Q., \& Viana, M. E. R. (2018). Atuação do enfermeiro na assistência ao pré-natal versus sífilis: uma revisão integrativa. Interfaces Científicas-Saúde e Ambiente, 6(2), 95-110.

de Freitas Aguiar, M. A. (2019). Psico-Oncologia: Assistência Humanizada eE Qualidade dDe Vida. Em de Freitas Aguiar, M. A. et al. Psico-oncologia: Caminhos de cuidado. Summus Editorial, 15-23.

Eichler, C., Pia, M., Sibylle, M., Sauerwald, A., Friedrich, W., \& Warm, M. (2015). Cognitive behavioral therapy in breast cancer patients-a feasibility study of an 8 week intervention for tumor associated fatigue treatment. Asian Pacific Journal of Cancer Prevention, 16(3), 1063-1067.

Faraji, J., Mahdavi, A., Samkhaniyan, E., Asadi, S. H., \& Dezhkam, N. (2015). A review of the effectiveness of cognitive-behavioral group therapy on the reduction of body image concern in patients with breast cancer. Journal of medicine and life, 8(Spec Iss 4), 82.

Fingeret, M. C., Teo, I., \& Epner, D. E. (2014). Managing body image difficulties of adult cancer patients: lessons from available research. Cancer, 120(5), 633-641.

Fonseca, R., \& Castro, M. M. (2016). A importância da atuação do psicólogo junto a pacientes com câncer: uma abordagem psico-oncológica. Psicologia e Saúde em debate, 2(Ed. Esp. 1), 54-72.

Gaspar, K. C. (2011). Psicologia hospitalar e a oncologia. Em Angerami, V. A. (Org.). Psicologia da saúde: Um novo significado para a prática clínica, 2. São Paulo: Cengage Learning, 79-126.

Greer, J. A., Traeger, L., Bemis, H., Solis, J., Hendriksen, E. S., Park, E. R., \& Safren, S. A. (2012). A pilot randomized controlled trial of brief cognitivebehavioral therapy for anxiety in patients with terminal cancer. The oncologist, 17(10), 1337.

Greer, J. A., Jacobs, J., Pensak, N., MacDonald, J. J., Fuh, C. X., Perez, G. K., \& Penedo, F. J. (2019). Randomized Trial of a Tailored Cognitive-Behavioral Therapy Mobile Application for Anxiety in Patients with Incurable Cancer. Oncologist, 24(8).

Ham, K., Chin, S., Suh, Y. J., Rhee, M., Yu, E. S., Lee, H. J., \& Chung, K. M. (2019). Preliminary Results From a Randomized Controlled Study for an AppBased Cognitive Behavioral Therapy Program for Depression and Anxiety in Cancer Patients. Frontiers in psychology, 10.

Instituto Nacional de Câncer José Alencar Gomes da Silva (2019). Estimativa 2020: Incidência de câncer no Brasil. Rio de Janeiro: Instituto Nacional do Câncer.

José, S. A. P., do Carmo, S. A., Vieira, L. R., Cardoso, L. C. P., Ferrari, S. R., Pinto, A. C. R., \& de Farias, P. V. A. (2020). A comunicação de notícias difíceis acerca da abordagem aplicada à oncologia: uma revisão bibliométrica. Research, Society and Development, 9(9), e569997570-e569997570.

Lourenção, V. C., dos Santos Junior, R., \& Luiz, A. M. G. (2009). Aplicações da Terapia Cognitivo-Comportamental em tratamentos de câncer. Revista Brasileira de Terapias Cognitivas, 5(2), 59-72.

Mann, E., Smith, M., Hellier, J., \& Hunter, M. S. (2011). A randomised controlled trial of a cognitive behavioural intervention for women who have menopausal symptoms following breast cancer treatment (MENOS 1): trial protocol. BMC cancer, 11(1), 44.

Mejía-Castrejón, J., \& Landa-Ramírez, E. (2014). Cognitive behavioural therapy for depression in multiple endocrine neoplasia type IIB: a 1-year follow-up. Case Reports, 2014, bcr2013202242.

Mendes, K. D. S., Silveira, R. C. D. C. P., \& Galvão, C. M. (2008). Revisão integrativa: método de pesquisa para a incorporação de evidências na saúde e na enfermagem. Texto \& contexto enfermagem, 17(4), 758-764.

Nieman, D. H., Sutterland, A. L., Otten, J., Becker, H. E., Drent, M. L., Van Der Gaag, M., ... \& De Haan, L. (2011). Treating prolactinoma and psychosis: medication and cognitive behavioural therapy. Case Reports, 2011, bcr0720103185.

Peron, N. B., \& Sartes, L. M. A. (2015). Terapia cognitivo-comportamental no hospital geral: revisão da literatura brasileira. Revista Brasileira de Terapias Cognitivas, 11(1), 42-49. 
Research, Society and Development, v. 10, n. 5, e33410514941, 2021

(CC BY 4.0) | ISSN 2525-3409 | DOI: http://dx.doi.org/10.33448/rsd-v10i5.14941

Poort, H., Onghena, P., Abrahams, H. J., Jim, H. S., Jacobsen, P. B., Blijlevens, N. M., \& Knoop, H. (2019). Cognitive Behavioral Therapy for TreatmentRelated Fatigue in Chronic Myeloid Leukemia Patients on Tyrosine Kinase Inhibitors: A Mixed-Method Study. Journal of Clinical Psychology in Medical Settings, 26(4), 440-448.

Rafihi-Ferreira, R. E., \& Soares, M. R. Z. (2012). Insônia em pacientes com câncer de mama. Estudos de Psicologia, 29(4), 597-607.

Souza, M. T. D., Silva, M. D. D., \& Carvalho, R. D. (2010). Integrative review: what is it? How to do it? Einstein, 8(1), 102-106.

Stang, L. D. G., \& Gomes, K. M. (2017). A importância da Terapia Cognitivo-Comportamental (TCC) no tratamento do paciente oncológico: Uma revisão não sistemática. Revista Interdisciplinar de Estudos em Saúde, 6(2).

Stefanopoulou, E., Yousaf, O., Grunfeld, E. A., \& Hunter, M. S. (2015). A randomised controlled trial of a brief cognitive behavioural intervention for men who have hot flushes following prostate cancer treatment (MANCAN). Psycho-Oncology, 24(9), 1159-1166.

Teo, I., Krishnan, A., \& Lee, G. L. (2019). Psychosocial interventions for advanced cancer patients: A systematic review. Psycho-oncology, 28(7), $1394-1407$.

Torres, A., Pereira, A., \& Monteiro, S. (2012). Terapia de grupo cognitivo-comportamental para sobreviventes de cancro da mama: descrição de um programa e avaliação preliminar da sua eficácia. Psychology, Community \& Health, 1, 300-312.

Thalén-Lindström, A., Larsson, G., Glimelius, B., \& Johansson, B. (2013). Anxiety and depression in oncology patients: A longitudinal study of a screening, assessment and psychosocial support intervention. Acta Oncologica, 52(1), 118-127.

Vilaça, A. P. O., \& Campos, E. M. P. (2020).; Técnicas cCognitivas eE cComportamentais nNo cCuidado aAo pPaciente oOncológico. Em Rodrigues, A. L. Psicologia da Saúde Hospitalar, Abordagem Psicossomática, 1. Manole, 113-125.

Wright, J. H.; Basco, M. R., \& Thase, M. E. (2008). Avaliação e Formulação. Em Wright, J. H., Basco, M. R., Thase, M. E. Aprendendo a Terapia CognitivoComportamental [recurso eletrônico]: Um guia ilustrado. Tradução de Mônica Giglio Armando. Dados eletrônicos. Artmed.

World Health Organization. (2020). WHO report on cancer: Setting priorities, investing wisely and providing care for all. Geneva: World Health Organization.

Whittemore, R., \& Knafl, K. (2005). The integrative review: updated methodology. Journal of advanced nursing, 52(5), 546-553. 